\title{
Tissue engineering tools for modulation of the immune response
}

\author{
Ryan M. Boehler, John G. Graham, and Lonnie D. Shea \\ Northwestern University, Evanston, IL, USA
}

BioTechniques 51:239-254 (October 2011) doi 10.2144/000113754

Keywords: Tissue engineering; immune modulation; immune response; biomaterials; gene delivery; cell transplantation

Supplementary material for this article is available at www.BioTechniques.com/article/113754

Tissue engineering scaffolds have emerged as a powerful tool within regenerative medicine. These materials are being designed to create environments that promote regeneration through a combination of: (i) scaffold architecture, (ii) the use of scaffolds as vehicles for transplanting progenitor cells, and/or (iii) localized delivery of inductive factors or genes encoding for these inductive factors. This review describes the techniques associated with each of these components. Additionally, the immune response is increasingly recognized as a factor influencing regeneration. The immune reaction to an implant begins with an acute response to the injury and innate recognition of foreign materials, with the subsequent chronic immune response involving specific recognition of antigens (e.g., transplanted cells) by the adaptive immune response, which can eventually lead to rejection of the implant. Thus, we also describe the impact of each component on the immune response, and strategies (e.g., material design, anti-inflammatory cytokine delivery, and immune cell recruitment/transplantation) to modulate, yet not eliminate, the local immune response in order to promote regeneration, which represents another important tool for regenerative medicine.

A foundational principle of regenerative medicine is creating an environment in which progenitor cells are able to develop into functional tissues in order to replace those lost to trauma or disease. The environment must present the appropriate combination of signals, which includes stimuli such as extracellular matrix proteins, growth factors and hormones, and mechanics. A central component of many approaches is the use of biomaterials, which create and maintain a defined space for tissue development and support cell growth. These materials can be employed as a vehicle for cell transplantation, or to recruit endogenous progenitor cells from the surrounding tissues. Additionally, these materials may serve as vehicles for the localized delivery of tissue inductive factors, delivered as proteins or as genes encoding for the factors. Materials, cells, proteins, and/or genes can be delivered in specific combinations in order to provide the microenvironment necessary to promote tissue regeneration for a variety of applications.

The immune response is a common issue in the use of biomaterials and the regeneration of all tissues. The process of transplanting cells, implanting biomaterial scaffolds, or delivering inductive factors can stimulate this immune reaction. The immune response fulfills multiple necessary purposes, such as removing cellular debris caused by the injury and limiting infection; however, the initial inflammatory response to the injury can lead to additional tissue damage (1), which can hinder regeneration. Preventing the infiltration of macrophages has been shown to lead to more extensive tissue damage and a decreased ability to regenerate (2). The development of strategies that harness the beneficial aspects of the immune response while limiting the potential deleterious aspects may be necessary to enhance regeneration.

This review describes the biomaterial systems common to regenerative medicine, and also their use in modulating the local immune response. We initially provide background on the inflammatory response to regenerative medicine strategies using biomaterials, drug and gene delivery, and cell transplantation, and subsequently describe strategies to employ these systems to modulate the inflammatory response.

\section{Tissue engineering tools and the inflammatory response}

The formation of functional tissues from endogenous or transplanted progenitor cells will require the presentation of a combination of signals that stimulate the appropriate cellular processes. Biomaterial implantation, drug and gene delivery, and cell transplantation alone typically have had limited efficacy in promoting regeneration; however, combining these strategies provides opportunities for greater control over the environment. The following sections describe the individual contributions of biomaterials, delivery systems, and cell transplantation for presenting biological signals, and also their potential to induce an immune response.

\section{Biomaterials}

Biomaterials are central to many strategies for regenerative medicine, with the scaffold functioning to create and maintain a space for tissue growth, provide mechanical stability, and support cell adhesion and migration. The range and variety of available materials has been the topic of several excellent reviews (3-4). Briefly, materials can be formed into hydrogels that have physical properties similar to that of native tissue. Natural materials (e.g., collagen, fibrin) intrinsically are bioactive, support cell adhesion, are degradable to allow for remodeling by infiltrating cells, and the degradation products 
may be bioactive (3). Hydrogels made from synthetic materials are being developed to provide a greater range of properties than is available with natural materials. The biological signals within these hydrogels can be more precisely controlled, with the ability to tune adhesion, degradation, and mechanics. Common alternative materials to hydrogels are porous polymer scaffolds, which typically have superior mechanical properties to hydrogels and are created to be highly porous to allow for cell infiltration and integration with the host tissue.

Biomaterials aim to create a local environment to promote tissue growth; however, both the injury incurred during the implantation process and the host inflammatory response to the implanted material can negatively impact this local environment. This response can lead to repair of the injury site through repopulation with granulation tissue that can result in fibrosis (5) while regeneration by local progenitor cells can produce fully functional tissue (6). An immune response immediately follows tissue injury, which can have a large impact on whether the wound is merely repaired or regenerated. The innate immune response to biomaterials has been reviewed elsewhere (5,7); a summary of the acute immune response is depicted in Figure 1. The primary insult causes activation of complement proteins by classical or alternative mechanisms (8) and activation of cellular pattern recognition receptors (PRRs) in response to presence of pathogens or cell damage (7), which leads to the initiation of inflammatory cytokine production (1) and subsequent chemokine (IL-8, MCP-1) recruitment of polymorphonuclear neutrophils (PMNs), monocytes, and fibroblasts to the injury site (9-10). PMNs infiltrate the wound first to remove pathogens and cellular debris present due to the injury and persist for only a few days $(1,11)$. PMNs eradicate pathogens using phagocytosis and release of reactive oxygen species (ROS) and inflammatory cytokines including IL-1 $\beta$, tumor necrosis factor- $\alpha$ (TNF- $\alpha$ ), and interferon- $\gamma$ $($ IFN- $\gamma)$, which can also cause secondary damage to the surrounding tissue (12). Monocytes differentiate into macrophages as they enter the injury site (1). The number of macrophages typically peaks around one week, yet they can persist in the injury site for months $(1,11)$. Macrophages also produce ROS and cytokines that can contribute to secondary damage (13). However, their presence is necessary for regeneration as they secrete growth factors (13) and phagocytose cell debris (14).

Implantation or injection of biomaterials, therapeutic factors, and/or foreign cells can intensify the inflammatory response by inducing a foreign body reaction and

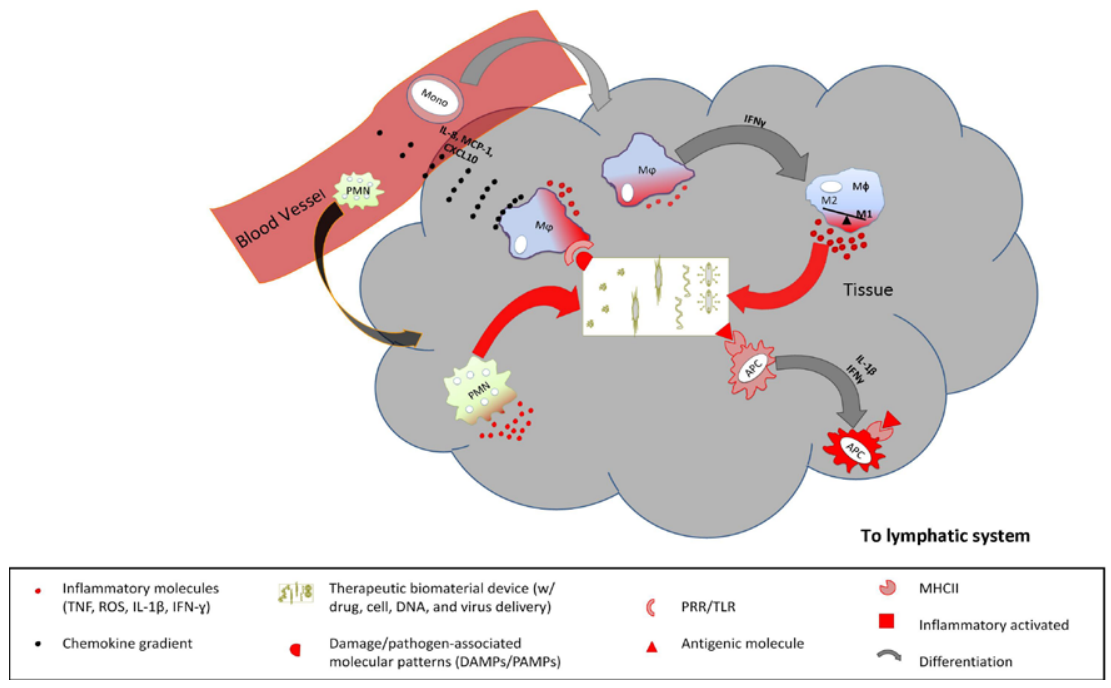

Figure 1. Acute Immune Response to Therapeutic Biomaterial Delivery System. The acute innate immune response is initiated by tissue injury and the presence of foreign materials. Resident cells (e.g. tissue macrophages $(M \phi)$ ) respond to increased damage/pathogen-associated molecular pattern molecules (DAMPs/PAMPs) by producing chemokines to recruit immune cells (PMNs and monocytes) from the blood stream. PMNs (the first immune cells recruited to the implant) produce large amounts of inflammatory molecules that can hinder regeneration. As monocytes enter the tissue, they differentiate into macrophages. Macrophages are induced to an M1 phenotype by inflammatory cytokines and contribute additional inflammatory molecules to the environment. Additionally, the adaptive immune response can be initiated during this acute phase by recognition of specific antigens on the viruses or cells that are delivered with the biomaterial. These factors activate APCs, which then transmigrate to the draining lymph node and spleen for antigen presentation.

introduce antigens to the site of injury. Blood-material interactions lead to protein deposition on the biomaterial forming a provisional matrix, which can affect subsequent leukocyte adhesion interactions. Thus, the chemical and physical properties of the surface of the biomaterial are largely responsible for the foreign body reaction propagated by infiltrating immune cells (5). Natural matrices can contain biological impurities and "non-self" signals resulting in increased inflammation at the implant site. While synthetic scaffolds can be produced without introducing these signals, synthetic polymers, their degradation products, or the associated provisional matrix can activate the complement cascade (8). Phagocytic cells are attracted to the implant by the chemokines released from the provisional matrix and surrounding cells. These cells adhere to the material, and if the material is large, may undergo a "frustrated phagocytosis" that can lead to increased secretion of inflammatory products (5).

The transition to the chronic inflammatory period (Figure 2) is signified by the departure of PMNs and some macrophages from the injury site, and infiltration of lymphocytes and plasma cells that are contributors to the adaptive immune response. Fibroblasts proliferate at the injury site and remodel the local extracellular matrix (ECM) to repair the wound. Whether this period results in tissue regeneration or scar formation is partially dependent on the duration of the chronic response that contributes to cytokine production and formation of granulation tissue. The remaining macrophages continue to produce cytokines, but the cytokines produced are dependent on their immune phenotype. The progression from an inflammatory (M1) phenotype toward a more regenerative/antiinflammatory (M2) phenotype correlates with a change in the cytokine secretion profile by $\mathrm{CD}^{+}$helper T (Th) cells from type I (Th1) to type II (Th2) that promotes resolution of inflammation (15). Macrophages have also been shown to adopt an M2 phenotype after phagocytosis of debris (14). A quick resolution (two weeks) to this chronic cellular presence at the tissue-material interface is often compatible with implant acceptance while persistence of a large immune cell presence often indicates infection and/or rejection of the implant (5).

\section{Cell transplantation}

Cell replacement therapy remains a powerful approach for numerous applications, with cells functioning to reconstitute a functioning tissue or to promote functional tissue regeneration. Cells are frequently delivered by direct injection, but biomaterials offer a vehicle for cell transplantation and provide a platform to enhance engraftment and function. Adult cells are often employed as they are differentiated and can restore tissue function (e.g., islets). Alternatively, stem and progenitor cells can be delivered as they can differentiate 
into multiple cell types within the developing tissue, differentiating along functionally necessary lineages, or may transiently provide trophic support that leads to tissue regeneration. In addition to cell type and differentiation state, a key consideration is the cell source. Some autologous cell therapies are available, such as for cartilage replacement. Patient specific mesenchymal stem cells can be isolated from peripheral blood and have been delivered for cardiac regeneration (16). However, for many applications, an autologous cell source is not available and allogeneic or xenogeneic sources are being developed. For example, islet transplantation is currently a treatment for type 1 diabetes mellitus (T1DM). In T1DM, the insulin producing $\beta$-cells are destroyed due to autoimmunity, resulting in the loss of control for blood glucose levels. Administering isolated pancreatic islets can provide a temporary cure and avoid the need for daily exogenous insulin (17). While research attempting to use widely available xenogeneic porcine islets is ongoing (18), the only current source for islets is cadaveric allogeneic donors.

Use of allogeneic or xenogeneic cells can induce an immune response in immunocompetent patients leading to rejection of transplanted cells (19). Rejection results from activation of the immune system due to surgical trauma initiating the inflammatory cascade and the presence of foreign

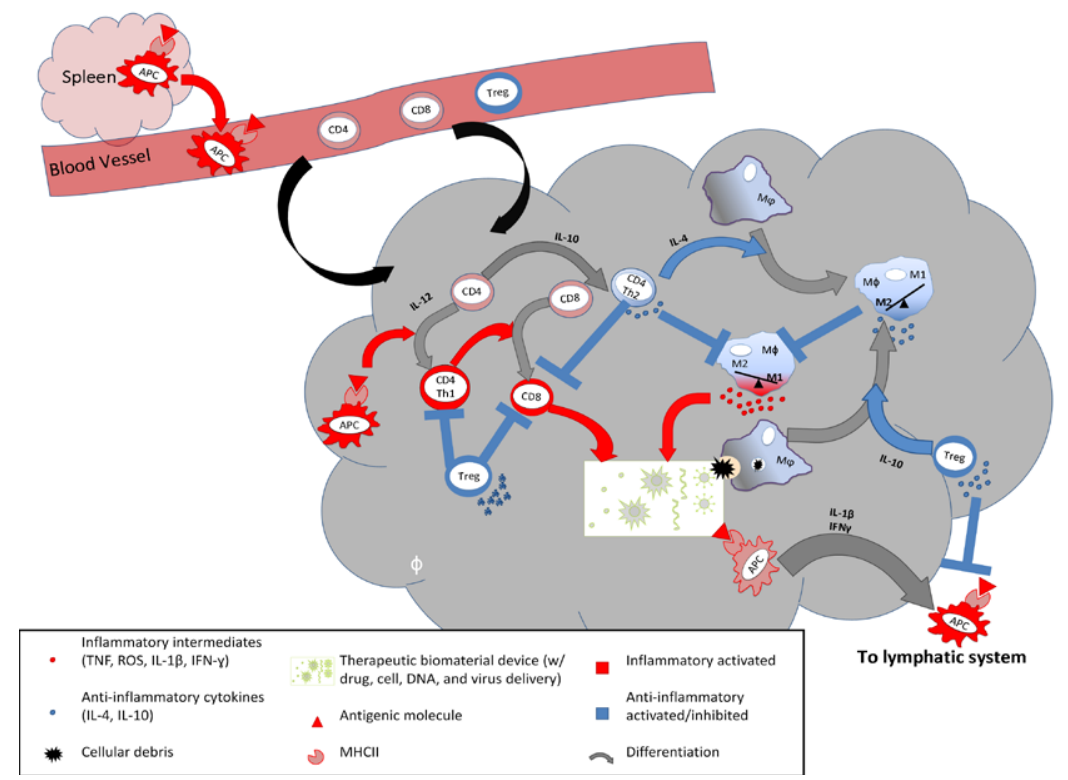

Figure 2. Chronic Immune Response to Therapeutic Biomaterial Delivery System. The chronic immune response is dominated by the adaptive immune response that is controlled by infiltrating $T$ cells. Th cells can be induced to either Th1 or Th2 cells, which can have functionally disparate immune responses. Activated APCs (from the spleen) or IL-12 can induce Th1 cells to activate CTLS and M1 macrophages, which potentially hinders regeneration and can lead to the destruction of transplanted cells. Th2 cells, which are induced by IL-10, inhibit Th1 processes and can alternatively induce an M2 macrophage phenotype, which may aid regeneration. Tregs also contribute to the inhibition of adaptive inflammatory processes (Th1 cells and activated APCs) and the promotion of M2 macrophages by producing IL-10. Additionally, phagocytosis of cellular debris may aid the natural progression of macrophages toward an M2 phenotype.

antigens associated with the cells promoting a more chronic adaptive response. The initial inflammatory cascade induces the release of pro-inflammatory cytokines (IL-1 $\beta, \mathrm{TNF}-\alpha$, and IFN- $\gamma$ ) that induce recruitment and activation of leukocytes, which produce ROS and additional cytokines that damage and destroy transplanted cells (20). IL-1 $\beta$ and cells (APCs) such as dendritic cells (DCs), initiating the adaptive immune response (21-22). These activated APCs migrate to the draining lymph node or spleen, where they present antigens from transplanted cells to Th cells. These Th cells subsequently proliferate and migrate to the graft where they release inflammatory cytokines and activate $\mathrm{CD}^{+}$cytotoxic $\mathrm{T}$ lymphocytes Ls), leading to graft destruction (23). Additionally, Th cells are responsible for initiating humoral immunity through $\mathrm{B}$ antibody production to target cellular response induced through the adminis tration of exogenous cells can ultimately lead to the dysfunction and destruction of transplanted cells.

\section{Delivery systems}

Biomaterial scaffolds can also function as vehicles for localized delivery of tissue inductive factors or genes encoding these factors. Numerous delivery strategies have been developed based on the physical properties of different materials, and approaches are generally categorized as either polymeric encapsulation or substrate immobilization (3). Sustained delivery from polymer substrates not only protects the protein or DNA from degradation, but also helps to maintain elevated levels of these factors within the extracellular environment by continual replacement of factors lost to clearance or degradation (3). Alternatively, substrate immobilization is based on the association or tethering of factors to the scaffold, using techniques such as adsorption, layer by layer assembly, or chemical conjugation. This approach places and maintains the factor directly in the cell microenvironment avoiding mass-transfer limitations, limiting potentially undesirable diffusion to distant sites. Immobilization can also regulate the distribution of factors and create patterns of expression (24). The following sections highlight protein and gene delivery capabilities, and the potential for immune response to these factors.

Protein delivery. Proteins initiate and control a variety of cellular processes in tissue formation, and their clinical utility continues to expand with advances in delivery systems and recombinant protein technology. Growth factors, cytokines, growth factor receptors, receptor antagonists, and monoclonal antibodies are currently being employed clinically. While many of these proteins exploit cell surface or extracellular targets, protein transduction domains are demonstrating promise for intracellular delivery (25). Localized delivery of tissue inductive factors from scaffolds can direct cell differentiation, promote cell survival, and induce organization or growth of tissue toward the desired regenerative state. In vitro studies of tissue formation on biomaterial scaffolds can be performed simply by adding growth factors to cell culture media; however, translation of these results in vivo requires delivery systems that provide these factors at the appropriate concentration and duration. Protein delivery has enhanced a number of processes in vivo, such as vascularization, development of differentiated phenotypes, and vaccination against specific antigens. In addition to releasing a single factor, combinations of factors have been delivered to target sequential cell processes, such as the initiation and maturation of blood vessels (26). Alternatively, factors delivered from defined regions of the scaffold have been shown to create defined concentration gradients, which can provide a directional signal to orient cellular processes and can enhance tissue formation (24). The sophistication of these delivery systems will be expected to impact numerous applications, and may also provide 
Table 1: Biomaterial Strategies for Immune Response Modulation

\begin{tabular}{|c|c|c|c|c|}
\hline Strategy & Factor & In vitro & In vivo & Ref. \\
\hline \multirow{4}{*}{ Material type } & Decrease hydrophobicity & $\begin{array}{l}\text { Decreased monocyte adhesion } \\
\text { and FBGC formation }\end{array}$ & $\begin{array}{l}\text { Decreased IL- } 6 \text { and TNF- } \alpha \\
\text { production }\end{array}$ & $45-47$ \\
\hline & $\begin{array}{l}\text { Remove cross-linking } \\
\text { procedures }\end{array}$ & & Increased M2 macrophage response & 48 \\
\hline & \multirow{2}{*}{$\begin{array}{l}\text { Choice of common } \\
\text { biomaterials }\end{array}$} & $\begin{array}{l}\text { Reduced DC maturation or } \\
\text { inflammatory cytokine production }\end{array}$ & & 49 \\
\hline & & $\begin{array}{l}\text { Non-adhesive substrates support } \\
\text { IL-10 production }\end{array}$ & & 50 \\
\hline \multirow{2}{*}{ Surface topography } & Aligned fibers & & Reduced capsule thickness & 51 \\
\hline & Creating microstructure & $\begin{array}{l}\text { Reduced monocyte fusion and } \\
\text { induced M1/M2 hybrid phenotype }\end{array}$ & Decreased capsule formation & $52-54$ \\
\hline \multirow{4}{*}{ Surface treatments } & Increase hydrophilicity & Limited DC maturation & & 55 \\
\hline & $\begin{array}{l}\text { Grafted microparticle } \\
\text { hydrogels }\end{array}$ & $\begin{array}{l}\text { Reduced protein adsorption and } \\
\text { monocyte adhesion }\end{array}$ & $\begin{array}{l}\text { Reduced leukocyte adhesion and } \\
\text { inflammatory cytokine production }\end{array}$ & 56 \\
\hline & Osteopontin coating & & $\begin{array}{l}\text { Reduced capsule thickness of } \\
\text { positively charged polymer surfaces }\end{array}$ & 57 \\
\hline & $\begin{array}{l}\text { Surfactant polymer } \\
\text { coating }\end{array}$ & & $\begin{array}{l}\text { Reduced fouling and platelet } \\
\text { adhesion }\end{array}$ & 58 \\
\hline
\end{tabular}

an enabling tool to dissect the molecular mechanisms in tissue development.

Formulations to provide sustained release must ensure the proteins are appropriately stabilized to retain bioactivity while avoiding eliciting an immune response. The presence of labile bonds or chemically reactive groups on the side chains can disrupt a protein's structure through aggregation or denaturation, which can lead to a loss of activity and immunogenicity (27). Proteins must be stabilized during encapsulation, storage, and subsequent administration. Stability can be maintained through controlling the process, such as by including surfactants, excipients, or antioxidants during the encapsulation process (27). Alternatively, stability may be maintained through PEGylation of the protein, which resists protein aggregation and adsorption. Covalently modified proteins can evade immune responses and may have an extended circulatory half-life in blood (28). However, PEGylation may also reduce protein uptake by target cells or immune cells (29), likely through steric hindrance and masking of surface charges.

Gene delivery. Delivery of non-viral or viral vectors that encode for therapeutic genes or induce RNA interference (RNAi) can enable sustained induction or knockdown of gene products, providing a versatile alternative to direct protein delivery for creating a microenvironment that promotes regeneration. Since each vector has similar physical properties independent of the gene sequence, this approach allows swapping of genes or even simultaneous delivery of multiple vectors that make one or more gene products. In this way, gene delivery can be used to stimulate local protein production capable of activating autocrine and paracrine loops that may play important roles in tissue development and physiology. Genes can be delivered in vivo that: (i) encode for desired protein(s), (ii) encode for transcription factors that regulate expression of the desired protein(s), or (iii) knock down production of proteins through RNAi. Relative to protein delivery, gene delivery can provide therapeutic concentrations of protein for long periods of time and target a variety of cell processes. DNA delivery can have a broader range of targets relative to protein delivery since the delivered DNA can target either intracellular or extracellular processes. The major challenge when using genes as opposed to directly delivering proteins is delivery efficiency; vectors must overcome several extracellular and intracellular barriers. Although viruses have evolved to efficiently deliver their cargo - if recognized by the immune system - they have the potential to be highly immunogenic. Non-viral strategies are generally considered safer than viral vectors; however, they lack the intrinsic efficiency of the viral systems.
Non-viral gene delivery. Plasmid can be delivered either naked or complexed with transfection reagents, such as cationic polymers or lipids termed polyplexes or lipoplexes, respectively. Upon internalization, plasmids must escape the intracellular endosome before degradation, and subsequently cross into the nucleus for expression. Plasmids are relatively stable and can be encapsulated within a range of biomaterials. Conversely, polyplexes and lipoplexes have the potential to aggregate and their encapsulation within materials can be challenging, though stabilizing formulations are being developed (30). An alternative to encapsulation is substrate immobilization, which has been accomplished by numerous strategies including: non-specific binding, specific binding, and layer-by-layer assembly $(3,31)$. These methods enable release to be tuned temporally and spatially. While temporally controlling the release of non-viral vectors has not had a significant impact on the extent or duration of gene expression (32), spatially controlled delivery has been employed in model systems of tissue formation, and the feasibility of in vivo spatially patterned gene delivery has been demonstrated (33-34).

Although the delivery of non-viral vectors is generally considered safe, these vectors do initiate an inflammatory response. Plasmids induce the non-specific activation of the inflammatory response similar to a bacterial infection through interactions with toll-like 
receptors (TLRs), a type of PRR present on APCs, monocytes, and B cells that nonspecifically binds to environmental antigens. Binding of plasmid to the TLRs activates the cell and induces the release of inflammatory cytokines that initiate the recruitment of additional inflammatory cells. The immune system can provide an additional barrier to efficient and sustained gene expression by recognition and clearance of vectors and transfected cells. Transgene expression may also be silenced, which is a function of the vector design. Specific DNA sequences, such as $\mathrm{CpG}$ motifs, may be recognized as foreign and can lead to gene silencing on timescales of days to weeks (35-36). Removal of this motif from the plasmid sequence has produced long-term expression (36). The promoter sequence is another consideration, which is exemplified by the commonly used cytomegalovirus (CMV) promoter. CMV activity is up-regulated by the transcription factor NF- $\kappa \mathrm{B}$, whose activity is dependent upon the inflammatory response (35). Long-term gene expression may also decline in response to immune system activation (35). Alternative promoters can provide more stable production, yet is dependent upon the tissue.

Viral gene delivery. Several types of viral vectors (e.g., adenovirus, lentivirus, adenoassociated virus) have been delivered from biomaterials as a means to obtain localized and efficient transduction (37). In selecting among the available vectors, transduction of target cells, duration of gene expression, and vector stability are considerations. The cells that can be targeted may be restricted to whether the vector can transduce dividing and non-dividing cells (38). Cell-specific promoters can be used if the gene delivery needs to be restricted to a single cell type. The duration of expression is dependent upon whether the vector integrates into the genome or remains episomal, and also clearance of transduced cells by the immune response (38). The vector's stability impacts the mechanisms by which it can be delivered. Adenoviral vectors have been encapsulated within biomaterials with retention of activity (39); however, the relative instability of some vectors has motivated the development of strategies in which the scaffolds are fabricated and vectors subsequently immobilized, which avoids virus exposure to the biomaterial processing conditions (37). Immobilization has been achieved using antibodies, biotin-avidin linkages, or through the presentation of plasma membrane components that interact with the virus (37).

The immune response elicited from the vector may influence gene expression. Innate immune response to the virus results from recognition of the capsid antigens on its surface
(38). The resulting inflammatory cytokine secretion may be less than that induced with non-viral vectors (40). Humoral antigen memory often limits virus delivery to a single administration; however, "stealth" viruses are being developed that can prevent recognition by the immune system (41). While cellular and humoral immunity have challenged the success of viral therapies in clinical trials (42), strategies are being researched to address these issues (43). The magnitude of the cellular response to the virus represents an obstacle to efficient gene expression due to the elimination of transduced cells by CTLs. Viruses that transduce APCs can produce a strong humoral-mediated due to direct antigen presentation (44).

Interestingly, delivery using biomaterials may facilitate evading the pre-existing immunity, ultimately enhancing transduction efficiency (37). Adenovirus delivery from biomaterials had a 45 -fold reduction in anti-adenovirus titers as compared with direct injection of adenovirus. Additionally, the material may shield the vector against neutralizing antibody or cellular immune responses, which may significantly reduce the quantities of viral vectors required for transduction in vivo, potentially enhancing the gene delivery efficiency.

\section{Modulation of inflammation}

The immune response has the potential to cause extensive secondary damage; however, more recent approaches have attempted to modulate the immune response in a manner than can more effectively promote regeneration at the site of injury. The following sections describe strategies employed to modulate the inflammatory response toward regeneration rather than repair.

\section{Biomaterials}

Reducing the immune response to implanted biomaterials may be achieved by choosing materials that are intrinsically immune-inert, or modifying material properties to prevent recognition by the immune system as summarized in Table 1. Biomaterial chemistry influences the proteins that adsorb, which mediates the interactions with immune cells and may lead to their activation. Generally, hydrophobic materials tend to enhance monocyte adhesion relative to hydrophilic materials leading to a local immune response at the implant site (45). Implantation of materials that are hydrophilic or neutral have decreased monocyte/macrophage adhesion and reduced foreign-body giant cell (FBGC) formation in vitro (46). However, adherent cells on hydrophilic or neutral biomaterials have been shown to produce a greater relative level of inflammatory cytokines (46). In vivo, materials with fewer FBGCs result in increased IL-6 and TNF- $\alpha$ production (47). Chemical crosslinking of natural ECMs is a common technique to increase stability of natural matrices, but it can impact the immune response. Acellular ECM scaffolds that are not chemically modified often result in an M2 macrophage response, while carbodiimide (CDI) cross-linking of scaffolds induces an M1 response (48). Some commonly-used materials can enhance DC maturation or inflammatory cytokine production (49), although the immune activation in response to these materials can be modified through delivery of cytokines or chemokines, or cell transplantation (discussed below). Additionally, adhesive substrates, such as collagen or vitronectin, have been shown to support Th1 DC responses in vitro, while serum-coated substrates induce IL-10 production and a Th2 response (50).

In addition to the material chemistry and subsequent protein adsorption, the surface topography and micron-scale architecture can modulate the interaction of immune cells that influences their activation. For example, scaffolds with an aligned fiber topography had a significantly reduced capsule formation and increased cell infiltration compared with scaffolds with randomly aligned fibers (51). Introducing microporosity to a scaffold is essential for tissue regeneration from infiltrating cells, but simply introducing micron-scale architecture to the surface of a biomaterial can affect its interactions with the immune system. The micro-architecture of the structure can reduce cell fusion relative to flat control surfaces (52) and has produced a macrophage phenotype that appears distinct from M1 and M2, while nano-architecture did not have an effect (53). The microarchitecture, independent of the chemistry, decreased capsule formation in vivo (54).

Beyond the material chemistry and architecture, the immune response to the material can be masked by surface treatments or coatings that provide a different surface chemistry for protein adsorption and recognition by the immune system. Treatment of the material to increase their hydrophilicity, which can impact the immune response, and has been used to limit dendritic cell maturation (55). Similarly, coatings have been employed to shield materials from protein adsorption and the subsequent immune response. Microparticle hydrogels have been grafted onto polymeric materials, which reduced protein adsorption and monocyte adhesion and resulted in reduced inflammatory cytokine levels after implantation (56). Osteopontin coatings on positively charged polymer surfaces have reduced capsule thickness around the implant (57). Treatment of materials with surfactant polymers reduced fouling and platelet adhesion (58). 
Table 2: Delivery System Strategies for Immune Response Modulation

\begin{tabular}{|c|c|c|c|c|}
\hline Strategy & Factor & In vitro & In vivo & Ref. \\
\hline Systemic delivery & Corticosteroid delivery & & Reduced leukocyte infiltration after SCI & 59 \\
\hline \multirow{4}{*}{$\begin{array}{l}\text { Localize immune cell } \\
\text { recruitment }\end{array}$} & Delivery of NSAIDs & & $\begin{array}{l}\text { Decreased PMN (not monocyte) recruitment } \\
\text { to sites of experimental arthritis }\end{array}$ & 9 \\
\hline & $\begin{array}{l}\text { Antibody neutralization of } \\
\text { CXCL10 }\end{array}$ & & $\begin{array}{l}\text { Reduced T cell recruitment and secondary } \\
\text { damage after SCl }\end{array}$ & 10 \\
\hline & $\begin{array}{l}\text { Adenoviral delivery of } \\
\text { IL-4 }\end{array}$ & & $\begin{array}{l}\text { Recruited Tregs to suppress inflammation in } \\
\text { EAE model }\end{array}$ & 60 \\
\hline & $\begin{array}{l}\text { Antibody blockage of } \\
\alpha 4 \beta 1 \text { integrin }\end{array}$ & & $\begin{array}{l}\text { Decreased early leukocyte (not chronic } \\
\text { monocyte) infiltration after } \mathrm{SCl}\end{array}$ & 61 \\
\hline \multirow{7}{*}{$\begin{array}{l}\text { Influence the } \\
\text { local immune cell } \\
\text { phenotypes }\end{array}$} & $\begin{array}{l}\text { Local delivery of } \\
\text { corticosteroids }\end{array}$ & & $\begin{array}{l}\text { Reduced inflammatory protein levels after } \\
\mathrm{SCl} \text { and has prevented fibrosis and } \mathrm{DC} \\
\text { maturation }\end{array}$ & $62-64$ \\
\hline & $\begin{array}{l}\text { Adenoviral delivery of } \\
\text { I } \mathrm{B} \alpha\end{array}$ & $\begin{array}{l}\text { Inhibited NF-KB- } \\
\text { induced inflammation }\end{array}$ & Inhibited NF-кB-induced inflammation & 65 \\
\hline & $\begin{array}{l}\text { Adenoviral delivery of } \\
\text { IL-1 and TNF- } \alpha \text { soluble } \\
\text { receptors }\end{array}$ & & $\begin{array}{l}\text { Decreased cartilage degradation and } \\
\text { leukocyte infiltration at sites of experimental } \\
\text { arthritis }\end{array}$ & 66 \\
\hline & \multirow{3}{*}{ Viral delivery of IL-10 } & & $\begin{array}{l}\text { Decreased inflammatory cytokines in wound } \\
\text { leading to regeneration }\end{array}$ & 67 \\
\hline & & & Prevented progression and relapse of EAE & 68 \\
\hline & & $\begin{array}{l}\text { Production of IL-10 } \\
\text { in transduced } \mathrm{C} 2 \mathrm{C} 12 \\
\text { cells }\end{array}$ & $\begin{array}{l}\text { Attenuated production of insulin auto- } \\
\text { antibodies and prevented T1DM }\end{array}$ & 69 \\
\hline & $\begin{array}{l}\text { Delivery of IL-10 } \\
\text { transduced fibroblasts }\end{array}$ & & $\begin{array}{l}\text { Transduced fibroblasts inhibited EAE while } \\
\text { IL-10 virus did not }\end{array}$ & 71 \\
\hline
\end{tabular}

Taken together, biomaterial chemistry and architecture are being manipulated to prevent up-regulation of inflammation, which may be a step toward promoting regeneration. However, these strategies may be insufficient for applications in which regeneration is blocked by the immune response (e.g., spinal cord injury) or the transplantation of allogeneic cells, which would typically be rejected by the host.

\section{Delivery systems}

Anti-inflammatory therapies aim to modulate the magnitude and diversity of immune cell responses, or alter the phenotype of the resident cells, leading to a resolution of inflammation as summarized in Table 2. Systemic delivery of anti-inflammatory cytokines has been employed to influence the immune cell response or phenotype, yet it has the potential to systemically deactivate the immune system, leaving the body vulnerable to infection. This systemic effect on the immune system may have unintended consequences on regeneration. Methylprednisolone (MP) can significantly reduce PMN and macrophage infiltration; however, functional outcomes have not resulted from this reduction in infiltration, which may be caused in part by the lack of beneficial long-term immune functions, such as debris removal by macrophages (59). Additionally, drug delivery systems are only therapeutic while the active agent is being released. Following depletion of the active agent, inflammatory responses can resume.

The beneficial effects of the immune response on regeneration may be retained using localized delivery systems, which do not impact the entire immune system and have the potential to selectively recruit specific immune cells or create a local anti-inflammatory microenvironment that influences the phenotype of infiltrating cells. Chemokines are responsible for inducing recruitment of immune cells to specific sites in the body. The chemokines CXCL8/IL-8, CCL2/MCP-1, and CXCL10 are crucial in the recruitment of PMNs, monocytes, and T cells respectively. Delivery of nonsteroidal anti-inflammatory drugs (NSAIDs) has reduced IL-8 and PMN levels while not significantly reducing MCP-1 and monocyte levels (9). Antibody neutralization of CXCL10 was shown to reduce $\mathrm{T}$ cell recruitment and secondary tissue damage (10). An alternative strategy has been to recruit immune suppressive cells. Inducing the expression of the anti-inflammatory cytokine IL-4 increases expression of CCL1, CCL17, and CCL22, which recruit regulatory $\mathrm{T}$ cells (Tregs) to suppress the inflammatory response (60). While chemokines are crucial for attracting immune cells to an inflamed site, integrin interactions are important for extravasation and retention at the injury site. Blocking these interactions has decreased early leukocyte infiltration and tissue oxidation after spinal cord injury while still allowing beneficial chronic macrophage infiltration (61). Controlling the numbers and types of immune cells at the implant site has the potential to reduce secondary tissue damage and promote regeneration.

The alternative strategy to promoting or inhibiting the recruitment of specific cell types is to influence the phenotype of the recruited immune cell population to further enhance regeneration. DCs mature to an inflammatory phenotype and macrophages can activate to an M1 phenotype in response to inflammatory stimuli. However, DCs and macrophages are able to attain an alternative activation phenotype in response to antiinflammatory stimuli such as IL-4, IL-10, transforming growth factor- $\beta 1$ (TGF- $\beta 1$ ), and corticosteroids (dexamethasone or DM, MP) leading to reduced inflammatory cytokine production and toleragenic responses (55). Localized delivery can quickly and directly influence the phenotype of infiltrating immune cells, preventing the up-regulation of inflammatory cytokines and thereby creating a localized regenerative microenvironment. One approach to inducing an anti-inflammatory immune phenotype is to directly deliver corticosteroids into the local microenvironment to promote regeneration. Conjugation of MP to polylactideco-glycolide (PLG) nanoparticles has reduced inflammatory protein levels (62). Release of 
Table 3: Cell Transplantation Strategies for Immune Response Modulation

\begin{tabular}{|l|l|l|l|}
\hline & Factor & In vivo & Ref. \\
\hline $\begin{array}{l}\text { Regulatory T cell delivery/ } \\
\text { induction }\end{array}$ & $\begin{array}{l}\text { Antigen-specific Tregs differentiated in vitro prolong islet graft survival } \\
\text { indefinitely in autoimmune disease }\end{array}$ & 78 \\
\hline $\begin{array}{l}\text { Mesenchymal stem cell delivery } \\
\text { delivery }\end{array}$ & $\begin{array}{l}\text { Suppress DC activation, prevent T cell, NK cell expansion } \\
\text { Sertoli cell delivery }\end{array}$ & $\begin{array}{l}\text { Prolongation of islet allografts when co-transplanted } \\
\text { Dendritic cell manipulation }\end{array}$ & $\begin{array}{l}\text { Ex vivo antigen-pulsed DCs provide antigen-specific protection when } \\
\text { reintroduced }\end{array}$ \\
\hline & EDCl-fixed splenocytes & $\begin{array}{l}\text { Splenocytes fixed with allo-antigen induce allogeneic tolerance in islet } \\
\text { transplantation }\end{array}$ & $\mathbf{7 1 ,}$ \\
\hline
\end{tabular}

DM from biomaterials has prevented fibrosis (63) and delayed DC maturation (64) in response to the implant. Improved understanding of the function of specific extracellular and intracellular proteins has also led to more targeted therapies. Targeted responses from proteins are exemplified by modulation of gene expression, sequestration of inflammatory cytokines, or delivery of anti-inflammatory cytokines. Up-regulated expression of $\mathrm{I} \kappa \mathrm{B} \alpha$ has directly inhibited inflammatory cytokine up-regulation by the transcription factor NF- $\kappa B$ (65). Delivery of soluble receptors for inflammatory cytokines has reduced inflammatory tissue damage (66). Delivery of anti-inflammatory cytokines has reduced inflammatory cytokine or autoantibody levels in animal models of wound healing, auto-immune encephalomyelitis (EAE), T1DM, and lupus (67-70) and has led to tissue regeneration instead of scar formation (67). However, direct delivery of retrovirus encoding for IL-10 failed to inhibit EAE, while IL-10 delivery from transduced fibroblasts succeeded in inhibition of EAE (71). Because therapies likely require the presentation of a combination of signals, immune cell transplantation has been investigated as a means to provide the variety of necessary signals.

\section{Cell transplantation}

A variety of cell types have been employed to modulate the immune response in order to promote the regeneration of tissues as summarized in Table 3. Mesenchymal stem cells (MSCs) can be isolated from bone marrow and expanded in vitro to develop into a range of tissues, such as bone, cartilage, and fat (72). The delivery of MSCs has been employed to enhance regenerative processes such as wound healing and neural repair (73), often through the secretion of trophic factors (74). However, these cells have also been reported to modulate the immune system in a range of applications, and this modulation may contribute to improved tissue formation. Upon reintroduction to the host, MSCs act on DCs and T cells to produce an immunoprivileged environment. MSCs prevent DC, T cell, macrophage, and PMN activation and proliferation through a variety of mechanisms including direct cellular interactions and secreting anti-inflammatory cytokines such as TGF-31 (75). Manipulating cells ex vivo and reintroducing the cells to the host provides a multimodal approach to controlling the immune system and providing immunoprotection of implanted materials and cells.

Transplantation of immune cells has been employed to modulate the immune response for applications in preventing graft rejection. The destruction of transplanted cells resulting from activated effector $T$ cells provides the largest barrier to engraftment, and these cell therapies target the inflammatory response that primes the immune cascade as well as directly targeting $T$ cells. Cells derived from immunoprivileged regions (e.g., Sertoli cells from the testis) have been delivered to promote cell engraftment and protect grafts against autoimmune and allogeneic rejection (76). These cells secrete a range of factors (TGF-31, IL-10, Fas ligand) that locally modulates immune cell function in addition to inducing Treg differentiation/expansion, which enhances immunoprotection.

Alternatively, immune cells such as DCs and Tregs may also be expanded ex vivo followed by re-administration into the host. DCs can be primed ex vivo in an antigen-specific manner by pulsing isolated DCs with antigen in the presence of antiinflammatory cytokines. These cells can then be reintroduced systemically or locally to provide protection from pre-defined antigens (77). Local administration provides the opportunity to co-localize immune cells with transplanted cells, thereby localizing the immune cells in the initial area of insult and reducing the number of cells necessary to transplant in comparison to systemic administration (78).

Similar to ex vivo priming of DCs, antigen-specific Tregs can be generated ex vivo by exposing Th cells to TGF- $\beta 1$ in the presence of APCs and antigen. Tregs normally comprise a small percentage of the $T$ cell population but these key regulators of the immune system can be expanded ex vivo, and have been used to protect transplanted cells from autoimmune destruction (78). Tregs can also be expanded in a non-antigenspecific manner via a similar in vitro culture system: Th cells in the presence of TGF- $\beta 1$ and stimulatory beads cause the conversion and expansion into CD4+CD25+FoxP3+ Tregs (78). Both non-specific and antigenspecific Tregs have been used to prevent the occurrence of autoimmune disease. Antigen-specific Tregs provide a tailored immunoprotection without affecting the immune system as a whole. Tregs, whether delivered locally or systemically, first home to the graft. Tregs can either remain in the graft, inducing further Treg differentiation, or move to draining lymph nodes or the spleen, where they interact with other $\mathrm{T}$ cells and APCs. In either of these locations, Tregs maintain immune cells in an inactivated state, secreting IL-10, TGF- $\beta 1$, and by direct interaction with immune cells (79).

Finally, cells have also been employed as antigen carriers to induce specific tolerance, either in the setting of autoimmune diseases (80) or to prevent rejection of transplanted cells (81-82). Donor derived splenocytes or splenocytes mixed with antigenic peptides were treated with the chemical crosslinker 1-ethyl-3-(3-dimethylaminopropyl) carbodiimide (ECDI). Splenocytes may directly present antigens to host $T$ cells, but elicit incomplete $T$ cell signaling which then silences subsequent $\mathrm{T}$ cell responses to the cognate antigen(s). Alternatively, splenocytes may be phagocytosed by host APCs, which then present the processed peptide antigens to host $\mathrm{T}$ cells in the context of host major histocompatibility complexes (MHCs). While direct presentation may play a role in tolerance induction, this mechanism is likely transient and does not explain the long term tolerance induced by this procedure. In contrast, indirect presentation by host regulatory APCs can produce long-term tolerance via the combined induction of anergy and activation of Tregs and is likely the predominant tolerance mechanism. However, the types of phagocytes and/or APCs involved, the signals to which they respond that determine tolerance versus immunity, the process of antigen processing and presentation, the circuits of interaction between APCs and various effector and regulatory populations, are largely unknown. 


\section{Conclusions}

Regenerative medicine offers tremendous potential for the replacement of functional tissues. Technologies based on biomaterials, drug, protein and gene delivery, and cell transplantation provide the fundamental tools, with the objective of creating an environment that supports the development of progenitor cells into functional tissues. While these technologies to control the environment are critical, the immune response is also an essential consideration. Each technological component has the potential to elicit an immune response that can derail regeneration and lead to fibrosis. However, appropriate application of these technologies has the potential to turn the immune response into an asset for regeneration, leading to the differentiation of cells toward a more regenerative and less inflammatory phenotype. Furthermore, recruitment or delivery of immune cells can be employed as a means to tolerize the host to prevent rejection of transplanted cells. Taken together, these technologies have broad implications for numerous applications of biomaterials in medicine, and also in emerging area of cellbased therapies.

\section{Acknowledgments}

The authors thank the reviewers for their helpful suggestions on this manuscript. We regret the inability to cite all the outstanding work in this area due to space constraints. Funding was provided by the National Institutes of Health (RO1EB005678, RO1EB9910, RO1 EB013198).

\section{Competing interests}

The authors declare no competing interests.

\section{References}

1. Donnelly, D.J. and P.G. Popovich. 2008. Inflammation and its role in neuroprotection, axonal regeneration and functional recovery after spinal cord injury. Exp. Neurol. 209:378-388.

2. Tidball, J.G. and M. Wehling-Henricks. 2007. Macrophages promote muscle membrane repair and muscle fibre growth and regeneration during modified muscle loading in mice in vivo. J. Physiol. 578:327-336.

3. De Laporte, L. and L.D. Shea. 2007. Matrices and scaffolds for DNA delivery in tissue engineering. Adv. Drug Deliv. Rev. 59:292-307.

4. Drury, J.L. and D.J. Mooney. 2003. Hydrogels for tissue engineering: scaffold design variables and applications. Biomaterials 24:4337-4351.

5. Anderson, J.M., A. Rodriguez, and D.T. Chang. 2008. Foreign body reaction to biomaterials. Semin. Immunol. 20:86-100.

6. Diegelmann, R.F. and M.C. Evans. 2004 Wound healing: an overview of acute, fibrotic and delayed healing. Front. Biosci. 9:283-289.
7. Jones, K.S. 2008. Effects of biomaterial-induced inflammation on fibrosis and rejection. Semin. Immunol. 20:130-136.

8. Nilsson, B., K.N. Ekdahl, T.E. Mollnes, and J.D. Lambris. 2007. The role of complement in biomaterial-induced inflammation. Mol. Immunol. 44:82-94.

9. Lopez-Armada, M.J., O. Sanchez-Pernaute, R. Largo, I. Diez-Ortego, I. Palacios, J. Egido, and G. Herrero-Beaumont. 2002. Modulation of cell recruitment by anti-inflammatory agents in antigen-induced arthritis. Ann. Rheum. Dis. 61:1027-1030.

10. Gonzalez, R., J. Glaser, M.T. Liu, T.E. Lane, and H.S. Keirstead. 2003. Reducing inflammation decreases secondary degeneration and functional deficit after spinal cord injury. Exp. Neurol. 184:456-463.

11. Beck, K.D., H.X. Nguyen, M.D. Galvan, D.L. Salazar, T.M. Woodruff, and A.J. Anderson. 2010. Quantitative analysis of cellular inflammation after traumatic spinal cord injury: evidence for a multiphasic inflammatory response in the acute to chronic environment. Brain 133:433-447.

12. Pineau, I. and S. Lacroix. 2007. Proinflammatory cytokine synthesis in the injured mouse spinal cord: multiphasic expression pattern and identification of the cell types involved. J. Comp. Neurol. 500:267-285.

13. Duffield, J.S. 2003. The inflammatory macrophage: a story of Jekyll and Hyde. Clin. Sci. (Lond.) 104:27-38.

14. Xu, W., A. Roos, N. Schlagwein, A.M. Woltman, M.R. Daha, and C. van Kooten. 2006. IL-10producing macrophages preferentially clear early apoptotic cells. Blood 107:4930-4937.

15. Martinez, F.O., A. Sica, A. Mantovani, and M. Locati. 2008. Macrophage activation and polarization. Front. Biosci. 13:453-461.

16. Yoon, Y.S., A. Wecker, L. Heyd, J.S. Park, T. Tkebuchava, K. Kusano, A. Hanley, H. Scadova, et al. 2005. Clonally expanded novel multipotent stem cells from human bone marrow regenerate myocardium after myocardial infarction. J. Clin. Invest. 115:326-338.

17. Shapiro, A.M., J.R. Lakey, E.A. Ryan, G.S. Korbutt, E. Toth, G.L. Warnock, N.M Kneteman, and R.V. Rajotte. 2000. Islet transplantation in seven patients with type 1 diabetes mellitus using a glucocorticoid-free immunosuppressive regimen. N. Engl. J. Med. 343:230-238.

18. Hering, B.J., M. Wijkstrom, M.L. Graham, M. Hardstedt, T.C. Aasheim, T. Jie, J.D. Ansite, M. Nakano, et al. 2006. Prolonged diabetes reversal after intraportal xenotransplantation of wild-type porcine islets in immunosuppressed nonhuman primates. Nat. Med. 12:301-303

19. Kirchhof, N., S. Shibata, M. Wijkstrom, D.M Kulick, C.T. Salerno, S.M. Clemmings, Y. Heremans, U. Galili, et al. 2004. Reversal of diabetes in non-immunosuppressed rhesus macaques by intraportal porcine islet xenografts precedes acute cellular rejection. Xenotransplantation 11:396-407.

20. Corbett, J.A., M.A. Sweetland, J.L. Wang, J.R. Lancaster, Jr., and M.L. McDaniel. 1993. Nitric oxide mediates cytokine-induced inhibition of insulin secretion by human islets of Langerhans. Proc. Natl. Acad. Sci. USA 90:1731-1735.

21. Fu, F., Y. Li, S. Qian, L. Lu, F. Chambers, T.E. Starzl, J.J. Fung, and A.W. Thomson. 1996. Costimulatory molecule-deficient dendritic cell progenitors (MHC class II+, CD80dim,
CD86-) prolong cardiac allograft survival in nonimmunosuppressed recipients. Transplantation 62:659-665.

22. Ito, T., M. Yang, Y.H. Wang, R. Lande, J. Gregorio, O.A. Perng, X.F. Qin, Y.J. Liu, and M. Gilliet. 2007. Plasmacytoid dendritic cells prime IL-10-producing $\mathrm{T}$ regulatory cells by inducible costimulator ligand. J. Exp. Med. 204:105-115.

23. Mellor, A.L. and D.H. Munn. 2008. Creating immune privilege: active local suppression that benefits friends, but protects foes. Nat. Rev. Immunol. 8:74-80.

24. Houchin-Ray, T., A. Huang, E.R. West, M. Zelivyanskaya, and L.D. Shea. 2009. Spatially patterned gene expression for guided neurite extension. J. Neurosci. Res. 87:844-856.

25. Gu, Z., A. Biswas, M. Zhao, and Y. Tang. 2011 Tailoring nanocarriers for intracellular protein delivery. Chem. Soc. Rev. 40:3638-3655.

26. Richardson, T.P., M.C. Peters, A.B. Ennett, and D.J. Mooney. 2001. Polymeric system for dual growth factor delivery. Nat. Biotechnol. 19:1029-1034.

27. Putney, S.D. and P.A. Burke. 1998. Improving protein therapeutics with sustained-release formulations. Nat. Biotechnol. 16:153-157.

28. Edwards, C.K., 3rd, S.W. Martin, J. Seely, O. Kinstler, S. Buckel, A.M. Bendele, M. Ellen Cosenza, U. Feige, and T. Kohno. 2003. Design of PEGylated soluble tumor necrosis factor receptor type I (PEG sTNF-RI) for chronic inflammatory diseases. Adv. Drug Deliv. Rev. 55:1315-1336.

29. Alemany, R., K. Suzuki, and D.T. Curiel. 2000. Blood clearance rates of adenovirus type 5 in mice. J. Gen. Virol. 81:2605-2609.

30. Lei, Y., S. Huang, P. Sharif-Kashani, Y. Chen, P. Kavehpour, and T. Segura. 2010. Incorporation of active DNA/cationic polymer polyplexes into hydrogel scaffolds. Biomaterials 31:9106-9116.

31. Jewell, C.M. and D.M. Lynn. 2008. Multilayered polyelectrolyte assemblies as platforms for the delivery of DNA and other nucleic acidbased therapeutics. Adv. Drug Deliv. Rev. 60:979-999.

32. Aviles, M.O., C.H. Lin, M. Zelivyanskaya, J.G. Graham, R.M. Boehler, P.B. Messersmith, and L.D. Shea. 2010. The contribution of plasmid design and release to in vivo gene expression following delivery from cationic polymer modified scaffolds. Biomaterials 31:1140-1147.

33. De Laporte, L., A. Huang, M.M. Ducommun, M.L.Zelivyanska, M.O. Aviles, A.F. Adler, and L.D. Shea. 2010. Patterned transgene expression in multiple-channel bridges after spinal cord injury. Acta Biomater. 6:2889-2897.

34. Phillips, J.E., K.L. Burns, J.M. Le Doux, R.E. Guldberg, and A.J. Garcia. 2008. Engineering graded tissue interfaces. Proc. Natl. Acad. Sci. USA 105:12170-12175.

35. Kako, K., M. Nishikawa, H. Yoshida, and Y. Takakura. 2008. Effects of inflammatory response on in vivo transgene expression by plasmid DNA in mice. J. Pharm. Sci. 97:30743083.

36. Hyde, S.C., I.A. Pringle, S. Abdullah, A.E. Lawton, L.A. Davies, A. Varathalingam, G. Nunez-Alonso, A.M. Green, et al. 2008. CpG-free plasmids confer reduced inflammation and sustained pulmonary gene expression. Nat. Biotechnol. 26:549-551.

37. Jang, J.H., D.V. Schaffer, and L.D. Shea. 2011 Engineering biomaterial systems to enhance viral vector gene delivery. Mol. Ther. 19:1407-1415.

38. Strayer, D.S. 1999. Viral gene delivery. Expert Opin. Investig. Drugs 8:2159-2172. 
39. Beer, S.J., C.B. Matthews, C.S. Stein, B.D. Ross, J.M. Hilfinger, and B.L. Davidson. 1998. Poly (lactic-glycolic) acid copolymer encapsulation of recombinant adenovirus reduces immunogenicity in vivo. Gene Ther. 5:740-746.

40. Sakurai, H., F. Sakurai, K. Kawabata, T. Sasaki, N. Koizumi, H. Huang, K. Tashiro, S. Kurachi, et al. 2007. Comparison of gene expression efficiency and innate immune response induced by Ad vector and lipoplex. J. Control. Release 117:430-437.

41. Croyle, M.A., N. Chirmule, Y.Zhang, and J.M. Wilson. 2001. "Stealth" adenoviruses blunt cell-mediated and humoral immune responses against the virus and allow for significant gene expression upon readministration in the lung. J. Virol. 75:4792-4801.

42. Mingozzi, F., N.C. Hasbrouck, E. BasnerTschakarjan, S.A. Edmonson, D.J. Hui, D.E. Sabatino, S. Zhou, J.F. Wright, et al. 2007. Modulation of tolerance to the transgene product in a nonhuman primate model of AAV-mediated gene transfer to liver. Blood 110:2334-2341.

43. Dai, Y., E.M. Schwarz, D. Gu, W.W. Zhang, N. Sarvetnick, and I.M. Verma. 1995. Cellular and humoral immune responses to adenoviral vectors containing factor IX gene: tolerization of factor IX and vector antigens allows for long-term expression. Proc. Natl. Acad. Sci. USA 92:1401-1405.

44. Bessis, N., F.J. GarciaCozar, and M.C. Boissier. 2004. Immune responses to gene therapy vectors: influence on vector function and effector mechanisms. Gene Ther. 11(Suppl 1):S10-S17.

45. Hezi-Yamit, A., C. Sullivan, J. Wong, L. David, M. Chen, P. Cheng, D. Shumaker, J.N. Wilcox, and K. Udipi. 2009. Impact of polymer hydrophilicity on biocompatibility: implication for DES polymer design. J. Biomed. Mater. Res. A 90:133-141.

46. Jones, J.A., D.T. Chang, H. Meyerson, E. Colton, I.K. Kwon, T. Matsuda, and J.M. Anderson. 2007. Proteomic analysis and quantification of cytokines and chemokines from biomaterial surface-adherent macrophages and foreign body giant cells. J. Biomed. Mater. Res. A 83:585-596.

47. Rodriguez, A., H. Meyerson, and J.M. Anderson. 2009. Quantitative in vivo cytokine analysis at synthetic biomaterial implant sites. J. Biomed. Mater. Res. A 89:152-159.

48. Brown, B.N., J.E. Valentin, A.M. StewartAkers, G.P. McCabe, and S.F. Badylak. 2009. Macrophage phenotype and remodeling outcomes in response to biologic scaffolds with and without a cellular component. Biomaterials 30:1482-1491.

49. Babensee, J.E. and A. Paranjpe. 2005. Differential levels of dendritic cell maturation on different biomaterials used in combination products. J. Biomed. Mater. Res. A 74:503-510.

50. Acharya, A.P., N.V. Dolgova, M.J. Clare-Salzler, and B.G. Keselowsky. 2008. Adhesive substratemodulation of adaptive immune responses. Biomaterials 29:4736-4750.

51. Cao, H., K. McHugh, S.Y. Chew, and J.M. Anderson. 2010. The topographical effect of electrospun nanofibrous scaffolds on the in vivo and in vitro foreign body reaction. J. Biomed. Mater. Res. A 93:1151-1159.

52. Chen, S., J.A. Jones, Y. Xu, H.Y. Low, J.M. Anderson, and K.W. Leong. 2010. Characterization of topographical effects on macrophage behavior in a foreign body response model. Biomaterials 31:3479-3491.
53. Paul, N.E., C. Skazik, M. Harwardt, M. Bartneck, B. Denecke, D. Klee, J. Salber, and G. Zwadlo-Klarwasser. 2008. Topographical control of human macrophages by a regularly microstructured polyvinylidene fluoride surface. Biomaterials 29:4056-4064.

54. Bota, P.C., A.M. Collie, P. Puolakkainen, R.B. Vernon, E.H. Sage, B.D. Ratner, and P.S. Stayton. 2010. Biomaterial topography alters healing in vivo and monocyte/macrophage activation in vitro. J. Biomed. Mater. Res. A 95:649-657.

55. Kou, P.M. and J.E. Babensee. 2011. Macrophage and dendritic cell phenotypic diversity in the context of biomaterials. J. Biomed. Mater. Res. A 96:239-260.

56. Bridges, A.W., N. Singh, K.L. Burns, J.E. Babensee, L. Andrew Lyon, and A.J. Garcia. 2008. Reduced acute inflammatory responses to microgel conformal coatings. Biomaterials 29:4605-4615.

57. Liu, L., G. Chen, T. Chao, B.D. Ratner, E.H. Sage, and S. Jiang. 2008. Reduced foreign body reaction to implanted biomaterials by surface treatment with oriented osteopontin. J. Biomater. Sci. Polym. Ed. 19:821-835.

58. Wang, S., A.S. Gupta, S. Sagnella, P.M. Barendt, K. Kottke-Marchant, and R.E. Marchant. 2009. Biomimetic fluorocarbon surfactant polymers reduce platelet adhesion on PTFE/ePTFE surfaces. J. Biomater. Sci. Polym. Ed. 20:619635.

59. Weaver, L.C., D. Gris, L.R. Saville, M.A. Oatway, Y. Chen, D.R. Marsh, E.F. Hamilton, and G.A. Dekaban. 2005. Methylprednisolone causes minimal improvement after spinal cord injury in rats, contrasting with benefits of an anti-integrin treatment. J. Neurotrauma 22:1375-1387.

60. Butti, E., A. Bergami, A. Recchia, E. Brambilla, U. Del Carro, S. Amadio, A. Cattalini, M. Esposito, et al. 2008. IL4 gene delivery to the CNS recruits regulatory $\mathrm{T}$ cells and induces clinical recovery in mouse models of multiple sclerosis. Gene Ther. 15:504-515.

61. Fleming, J.C., F. Bao, Y. Chen, E.F. Hamilton, J.K. Relton, and L.C. Weaver. 2008. Alpha4beta1 integrin blockade after spinal cord injury decreases damage and improves neurological function. Exp. Neurol. 214:147-159.

62. Chvatal, S.A., Y.T. Kim, A.M. Bratt-Leal, H. Lee, and R.V. Bellamkonda. 2008. Spatial distribution and acute anti-inflammatory effects of Methylprednisolone after sustained local delivery to the contused spinal cord. Biomaterials 29:1967-1975.

63. Blanco, E., B.D. Weinberg, N.T. Stowe, J.M. Anderson, and J. Gao. 2006. Local release of dexamethasone from polymer millirods effectively prevents fibrosis after radiofrequency ablation. J. Biomed. Mater. Res. A 76:174-182.

64. Norton, L.W., J. Park, and J.E. Babensee. 2010. Biomaterial adjuvant effect is attenuated by antiinflammatory drug delivery or material selection. J. Control. Release 146:341-348.

65. Wilson, H.M., S. Chettibi, C. Jobin, D. Walbaum, A.J. Rees, and D.C. Kluth. 2005. Inhibition of macrophage nuclear factorkappaB leads to a dominant anti-inflammatory phenotype that attenuates glomerular inflammation in vivo. Am. J. Pathol. 167:27-37.

66. Ghivizzani, S.C., E.R. Lechman, R. Kang, C. Tio, J. Kolls, C.H. Evans, and P.D. Robbins. 1998. Direct adenovirus-mediated gene transfer of interleukin 1 and tumor necrosis factor alpha

\section{Glass \\ Volumatrio Plastio Labwarol BRAND}

\section{西}

- Accurate:

Class A and

Class B versions

available

- Safe \&

Economical:

Volumetric

plasticware resists

breakage, saves

on replacement

costs

- Versatile:

Plastic options

for chemical

compatibility and

trace analysis

BrandTech offers a full line of volumetric plastic labware \& general laboratory plastics. Available through leading lab

dealers!
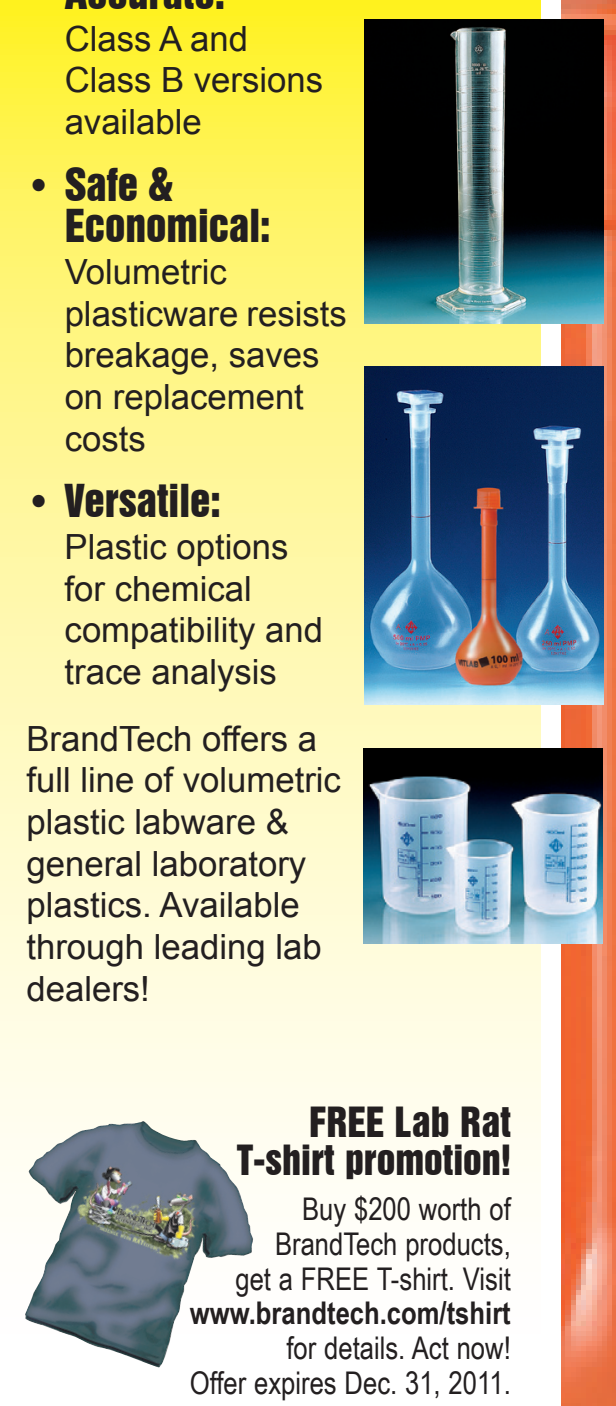

Lab Rats Trust BrandTech!

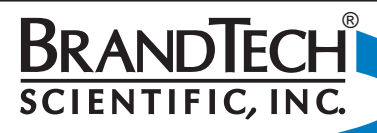

Toll Free (888) 522-2726 www.brandtech.com 
soluble receptors to rabbit knees with experimental arthritis has local and distal anti-arthritic effects. Proc. Natl. Acad. Sci. USA 95:4613-4618.

67. Peranteau, W.H., L. Zhang, N. Muvarak, A.T. Badillo, A. Radu, P.W. Zoltick, and K.W. Liechty. 2008. IL-10 overexpression decreases inflammatory mediators and promotes regenerative healing in an adult model of scar formation. J. Invest. Dermatol. 128:1852-1860.

68. Cua, D.J., B. Hutchins, D.M. LaFace, S.A Stohlman, and R.L. Coffman. 2001. Central nervous system expression of IL-10 inhibits autoimmune encephalomyelitis. J. Immunol. 166:602-608.

69. Goudy, K., S. Song, C. Wasserfall, Y.C. Zhang, M. Kapturczak, A. Muir, M. Powers, M. ScottJorgensen, et al. 2001. Adeno-associated virus vector-mediated IL-10 gene delivery prevents type 1 diabetes in NOD mice. Proc. Natl. Acad. Sci. USA 98:13913-13918.

70. Prud'homme, G.J. 2000. Gene therapy of autoimmune diseases with vectors encoding regulatory cytokines or inflammatory cytokine inhibitors. J. Gene Med. 2:222-232.

71. Croxford,J.L., M. Feldmann, Y. Chernajovsky, and D. Baker. 2001. Different therapeutic outcomes in experimental allergic encephalomyelitis dependent upon the mode of delivery of IL-10: a comparison of the effects of protein, adenoviral or retroviral IL-10 delivery into the central nervous system. J. Immunol. 166:4124-4130.

72. Marion, N.W. and J.J. Mao. 2006. Mesenchymal stem cells and tissue engineering. Methods Enzymol. 420:339-361.

73. Berman, D.M., M.A. Willman, D. Han, G. Kleiner, N.M. Kenyon, O. Cabrera, J.A. Karl,

\section{Protocol Guide and Poster Hall Resources}

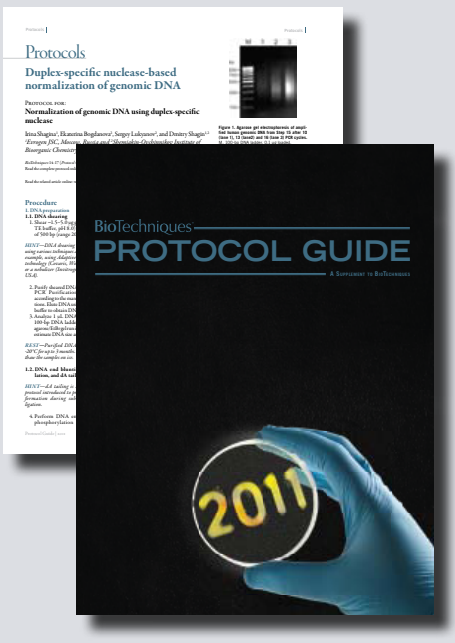

Poster Hall Topics Include:

Antibody-based Technologies

- Cell Signaling

- FFPE

High-throughput Screening

- Microarray Analysis

- Plasmid DNA Purification

- Transfection

www.BioTechniques.com/Posters

\section{Two Unique Life Science Resources}

Protocol Guide Topics Include:

Biomarker Validation

Cell Culture

Cytometry

DNA/RNA Isolation/Purification

- Epigenetics

- Gene Expression and Transfer

Microscopy

- PCR

www.BioTechniques.com/Protocols

\section{BioTechniques}

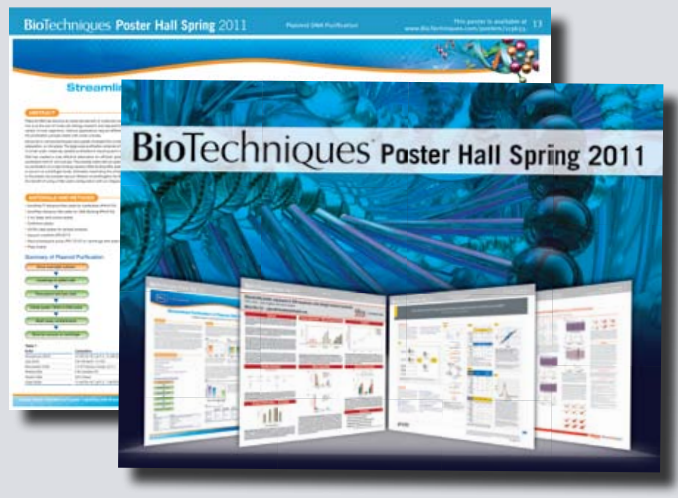

R.W. Wiseman, et al. 2010. Mesenchymal stem cells enhance allogeneic islet engraftment in nonhuman primates. Diabetes 59:2558-2568.

74. Bartholomew, A., D. Polchert, E. Szilagyi, G.W. Douglas, and N. Kenyon. 2009. Mesenchymal stem cells in the induction of transplantation tolerance. Transplantation 87:S55-S57.

75. Di Nicola, M., C. Carlo-Stella, M. Magni, M. Milanesi, P.D. Longoni, P. Matteucci, S. Grisanti, and A.M. Gianni. 2002. Human bone marrow stromal cells suppress T-lymphocyte proliferation induced by cellular or nonspecific mitogenic stimuli. Blood 99:3838-3843.

76. Dufour, J.M., S.J. Lord, T. Kin, G.R. Rayat, D.E. Dixon, R.C. Bleackley, G.S. Korbutt, and R.V. Rajotte. 2008. Comparison of successful and unsuccessful islet/Sertoli cell cotransplant grafts in streptozotocin-induced diabetic mice. Cell Transplant. 16:1029-1038.

77. Garrovillo, M., A. Ali, and S.F. Oluwole. 1999 Indirect allorecognition in acquired thymic tolerance: induction of donor-specific tolerance to rat cardiac allografts by allopeptide-pulsed hos dendritic cells. Transplantation 68:1827-1834.

78. Luo, X., K.V. Tarbell, H. Yang, K. Pothoven, S.L. Bailey, R. Ding, R.M. Steinman, and M. Suthanthiran. 2007. Dendritic cells with TGF-beta1 differentiate naive CD4+CD25- T cells into islet-protective Foxp3+ regulatory $\mathrm{T}$ cells. Proc. Natl. Acad. Sci. USA 104:2821-2826.

79. Zhang, N., B. Schroppel, G. Lal, C. Jakubzick, X. Mao, D. Chen, N. Yin, R. Jessberger, et al. 2009. Regulatory T cells sequentially migrate from inflamed tissues to draining lymph nodes to suppress the alloimmune response. Immunity 30:458-469.

80. Kennedy, M.K., L.J. Tan, M.C. Dal Canto, V.K. Tuohy, Z.J. Lu, J.L. Trotter, and S.D. Miller. 1990. Inhibition of murine relapsing experimental autoimmune encephalomyelitis by immune tolerance to proteolipid protein and its encephalitogenic peptides. J. Immunol. 144:909-915

81. Luo, X., K.L. Pothoven, D. McCarthy, M. DeGutes, A. Martin, D.R. Getts, G. Xia, J He, et al. 2008. ECDI-fixed allogeneic splenocytes induce donor-specific tolerance for long-term survival of islet transplants via two distinct mechanisms. Proc. Natl. Acad. Sci. USA 105:14527-14532.

82. Kheradmand, T., S. Wang, R.F. Gibly, X Zhang, S. Holland, J. Tasch, J.G. Graham, D.B Kaufman, et al. 2011. Permanent protection of PLG scaffold transplanted allogeneic islet grafts in diabetic mice treated with ECDIfixed donor splenocyte infusions. Biomaterials 32:4517-4524.

Received 01 September 2001; accepted 12 September 2011.

Address correspondence to Lonnie Shea, Department of Chemical and Biological Engineering, Northwestern University, Evanston, IL 60208, USA. Email: 1-shea@northwestern.edu.

To purchase reprints of this article, contact: biotechniques@fosterprinting.com

www.BioTechniques.com/Subscribe 